\title{
07- INQUÉRITO
}

Tipo de inquérito: (DI)

doc.: non:: età

inf.: età $(++)$ sessantanove anni sesso $(+)$ maschile genitori $(+)$ di origine veneta stato civile $(++)$ celibe luogo di nascita $(t)$ provincia di Vicenza luogo di studio luoghi

05 di studio Bassano del Grappa $(++)$ Piacenza $(++)$ Como $(+)$ e Roma data dell'arrivo in Brasile millenovecentoecinquantadue tempo di residenza in San Paolo ( ) di San Paolo (+) quindici anni (+) e poi nei dintorni di San Paulo

10 doc.: (ora) come mai lei è venuto in Brasile?

inf.: sono venuto in Brasile (+) PER COMpiere una missione:: mi han chiesto i superiori se ero disposto: di venire in Brasile $(+)$ per dedicarmi a immigranti italiani $(++)$ questa era (la) nostra missione $(+)$ io ho detto "proviamo $(++)$

15 vediamo se $(+)$ se mi troverò bene se sarà" doc.: inf.: I in quell'epo/ [ "possibile" doc.: in quell'epoca era possibile che lei potesse andare da un'altra parte che non fosse il Brasile?

20 inf.: sì

doc.: lei ha potuto scegliere?

inf.: sì ahn:: si può dire che io ho potuto scegliere nel senso che $(+)$ ((tosse)) ahn:: avendo la nostra congregazione avendo la nostra congregazione miSSIOni in tutte le parti del mondo dove c'è: $(+)$ l'emigrazione italiana soprattutto dove

c'è l'emigrazione italiana Stati Uniti Australia eh:: (+) ah Europa

eh: Canada ( + ) e:: Argentina eh: Cile $(+)$ Peru eccetera e ci sarebbe

stata questa possibilità di scegliere: un altro paese $(+)$ difatti $(++)$ dei miei:: colleghi: siamo stati ordinati in diciotto eh:: solo io sono venuto in Brasile

doc.: e come era la situazione dell'Italia quando lei è venuto via? inf.: è quando sono venuto via $(+)$ tutti noi sappiamo $(+)$ che l'Italia si trovava in condizioni MOLto difficili perché 
$(+)$ era appena finita la guerra $(+)$ una guerra $(++)$ ahn:: che ha deva/ devastatrice $(+)$ che ha causato $(+)$ molti danni molte morti $(+)$ sacrifici dolori non solo in Italia ma in tutta l'Europa $(+)$ e quindi possiamo immaginarci $(+)$ appena finita guerra eh:: come l'Italia

40 si: si trovasse in mezzo a mille difficoltà $(+)$ eh:: provazioni $(+)$ e di fatto uhn:: eh: uhn: appena terminata la guerra mo/ MOLti hanno cercato dimenticare questo periodo triste della guerra $(+)$ e del fascismo e trovare posti più sicuri $(+)$ eh: più:: ahn: $(+)$ più ahn:: 45 più pa/ pacifici e $(+)$ con la possibilità di di offrire alle nuove generazioni eh: : migliori prospettive $(+)$ e allora si può dire che questa sia stata l'ultima l'ultima $(+)$ eh:: leva di di immigranti italiani che son usciti dall'Italia in questo dopo guerra $(+)$ eh:: terminata la 50 guerra in millenovecentoequarantacinque non proprio subito subito dopo la guerra ma qualche anno dopo vedendo che era difficile trovare un lavoro eh:: vedendo che:: che:: insomma era:: sembrava quasi impossibile la ricostruzione tutto era distrutto e molti:: eh:: molti 55 molti emigranti uscivano ahn:: in quegli anni ahn:: eh: del dopo guerra si può dire che questo questo periodo dell'ultima emigrazione italiana sia durata più o meno fino al millenovecentossessanta sessantacinque è possibile poi si è chiusa $(+)$ e quindi era veramente un 60 momento quando io sono stato ordinato eh:: invitato a questa missione in Brasile era un momento forte di: di: emigrazione di problemi emigratori di gente che usciva di: (+) eh:: insomma di speranze nuove anche eh:: di entusiasmo per questi nuovi luoghi eh: per molti 65 sconosciuti ma che sempre: suscitavano: interesse entusiasmo soprattutto soprattutto sempre è stato un sogno in Europa di: di delle AMEriche sì di andare in AMErica per fare fortuna in AMErica perché l'America è GRAnde è bella eccetera e così via $(+)$ e molti pure 70 sapendo che c'era questa questa distinzione tra America del Sud ((rumori)) e America del Nord ((ride)) ben differenti una dell'altra (non è vero?) ((rumore)) 
doc.: ok

e lei è qui dopo tanto tempo che è in Brasile considerando le esperienze che lei ha vissuto ha vissuto se potesse tornare indietro in quel momento di fare la scelta la scelta sceglierebbe ancora il Brasile?

inf.: eh: uhn:: può essere che sì ma $(++)$ ho:: dei dubbi ((ride)) ((ridendo)) ho dei dubbi forse

80 doc.: e (su) quali aspetti ci sono stati inf.:

doc.: delle delusioni:: o delle::

inf.: sì perché: $(+)$ ho visto che:: $(+)$ ho visto che:: molti sogni molte speranze $(+)$ uhn:: $(+)$ non tan/ non tanto per ME perché $(+)$ la nostra missione non è quella:: eh: di:: sogni speranze materiali la nostra missione è essenzialmente spirituale $(+)$ e però noi ci ci identifichiamo $(+)$ con le con i nostri co/ connazionali con (la le) nostre comunità sentiamo (t) CHE:: molti sono rimasti disillusi e quindi: noi assumiamo pure noi queste disillusioni queste speranze perdute $(+)$ eh:: ormai $(+)$ forse anche $(++)$ non più possibile a essere eh:: a essere riaccesa la:: la fiamma di queste speranze di questi sogni di di futuro in:: questo senso voglio dire $(+)$ che: c'è

95 stato un grande progresso dapperTUtto anche qui in Brasile c'è stato molto progresso dal cinquantadue fin'ora in quarantanni però questo progresso in: altre parti ha: beni/ ha benificiato tutte le classi qui in Brasile è stato favorevole: più a una elite che a tutto un popolo $(+)$ e difatto e difatto io ho l'impressione che per il popolo: $(+)$ ah: semplice per il popolo $(+)$ ahn:: così: eh: $(+)$ comune $(+)$ sia sia vive/ viveva meglio questo popolo $(+)$ popolino come si costuma si suol dire viveva meglio in quel tempo nel cinquanta

105 quando io sono arrivato che adesso: almeno: c'erano delle cose essenziali c'erano per tutti $(+)$ c'era: più tranquilità più:: più semplicità più:: $(+)$ più felicità forse anche ( ) era più felice

doc.: perché c'era anche la speranza

110 inf.: èh sì perché c'era anche la speranza c'era il necessario non mancava per nessuno $(+)$ c'era più rispetto c'era più moralità $(+)$ c'era più:: giustizia nonostante le 
differenze sociali che sono sempre state un grosso un grosso problema non solo qui in Brasile ma per in molte parti del mondo anche in Italia stessa eh:: non c'erano le violenze che ci sono adesso $(+)$ non c'erano le le ristrettezze le:: ansietà uhn: $(+)$ e tanti problemi sono che sono sono sorti dopo insieme al al progresso l'industrializzazione è stata troppo veloce $(+)$ il popolo il popolino non era preparato $(+)$ la natura ci insegna a non fare salti strappi bisogna andare gradativamente $(+)$ nella ((squilla il telefono)) l'evoluzione delle cose non può avvenire così da un momento: per altro abbiamo qui in San Paolo un esempio classico di una città che:: è sorta è cresciuta disordinatamente ((rumore)) ogni giorno ne sentiamo le conseguenze

doc.: allora e lei:: ben lei più o meno ci ha detto già un po' com'era il Brasile in quell'epoca le differenze del Brasile di quell'epoca e del Brasile d'oggi ((qualcuno lo chiama, si interrompe l'intervista)) pensa di potere ritornare in Italia svolgendo la sua::attività? inf.: [ beh questo questo è anche:: (+) una domanda ahn: che può:: diciamo $(+)$ che può interessare su un punto di vista anche 135 psicologico perché $(+)$ da quanto io ho detto potrebbe sembrare che $(++)$ potessi desiderare eh:: un un altro luogo eh sì eh:: si ho dei motivi di delle critiche da fare sul Brasile eh:: sulle difficoltà che ci sono $(+)$ sulle mie su certe disillusioni eh:: speranze

140 perdute come ho detto prima $(+)$ però $(+)$ vorrei anche sottolineare gli aspetti positivii ((ride)) èh di questa: diciamo di questa esperienza che fin dal principio mi è piaciuto il Brasile mi è piaciuto il POpolo brasiliano $(+)$ ho trovato diffi/ difficoltà per il clima $(+)$ per il clima eh:: eh:: io preferirei clima freddo piuttosto che il clima qua così: eh:: tropicale beh qui a San Paolo ancora non tanto ma uhn:: fuori di San Paolo eh::: si trova facilmente ((suona il campanello)) eh:: difficoltà (suona nuovamente il campanello) abbiamo ( ) difficoltà per

150 causa del clima anche doc.: lei non ha avuto possibilità per esempio di:: di stare lì al sud del Brasile dove più o meno il clima sarebbe 
inf.:

[ Sİ ci

sa/ vi sarebbe anche questa possibilità di stare di stare più al sud eh: di San Paolo verso verso: eh:: Rio Grande del Sud no?

doc.: èh

inf.: anche lì abbiamo molte case molte parrocchie Rio Grande del Sud ci sarebbe anche questa possibilità ma dico ((tosse)) ci sono molti ah molti aspetti positivi ehn:: per il clima c'è a molti piace moltissimo molti europei preferiscono qui eh:: in Brasile piuttosto che in Europa e per sfuggire soprattutto $i$ i rigorosi inverni eh: che sono che sono veramente sono veramente eh: rigoROsi ehn:

165 e fanno soffrire le persone che temono il freddo $(+)$ eh:: ma più che altro il popolo brasiliano mi è piaciuto $(+)$ e:: e poi:: ci si ci si affeziona dopo tanti anni ci si abitua si finisce per abituarsi e quindi voglio dire che adesso adesso dopo dopo quaranT'Anni eh: forse: io temo

170 che: eh:: troverei difficoltà a adattarmi nuovamente $(+)$ in Europa o anche $i /$ in altri posti a una certa età è:: più difficile cambiare (di) quando si è giovani vero? a una certa età $(+)$ e quindi $(+)$ tutto sommato io preferisco rimanere qui in Brasile stesso $(+)$ e poi se è

175 questa la missione se ho detto di sì a a fin $\mathrm{dal} /$ dall'inizio di questa missione e se questa (insomma) e se questo è stato scritto nei disegni di Dio eh:: noi cerchiamo di:: interpretare nei nei segni nei vari segni che lui ci manda questa sua volonTA e allora eh:: mi pare

180 che sia coerente a: andare andare verso questa volontà fino alla fine e: cercare di compiere nel::: meglio/ migliore modo possibile questa missione che lui ci ha affidato e:: uhn: integralmente eh: fino in fondo $(+)$ come lui ci ha amato fino alla fine e e: il il modo

185 credo che sia il modo migliore per ricambiare questo amore eh che lui ci ha ci ha dato per primo eSEmpio e:: ricambiare questo amore con la stessa generosità e:: $e$ soprattutto con con fiducia in lui che certamente $(+)$ non si lascerà vincere in generosità

190 doc.: lei ha detto per esempio le difficoltà che si presentavano inf.: doc.: che lui si è ricordato così 
inf.: [ èh:

doc.: in particolare è stato il CLIma diciamo

195 inf.: beh sì

doc.: [ èh

inf.: il clima è una delle difficoltà che ho trovato

doc.: [ una difficoltà

inf.: è stato il clima specialmente:: qui a San Paolo non tanto

200 ma quando sono stato trasferito a: Rio de Janeiro (+) mi ricordo che Rio de Janeiro è una città bellissima è una città meravigliosa come dicono e lo è anche pure senza dubbio ma: ma una delle:: TRIsti ricordi che mi sono rimasti nelle mie esperienze di otto anni a Rio de Janeiro è

205 stata è stato il caldo il clima la difficoltà di adattarmi a questo a questo clima

doc.: e i contatti iniziali con i brasiliani com'è stato come sono stati

inf.:

i contatti i contatti iniziali con i brasiliani sono stati buoni

doc.: secondo

inf.: [ sono stati molto buoni

doc.: lei

inf.: [ sì

215 doc.: i brasiliani sono

inf.: [ sì

doc.: della gente accogliente

inf.: [ sì sì sì molto buoni

doc.: parliamo un po' torniamo un po' indietro pensiamo

220 parliamo un po' di quel momento lì quando lei si è reso conto che era il sacer/ sacerdozio la sua missione uhn? quando è stato? ancora adolescente:?

inf.: beh sì io sono entrato in seminario a tredici anni doc.: $a h$

225 inf.: in quel tempo era così $(+)$ e il se/ il seminario era pieno a Bassano del Grappa in provincia di Vicenza e $(+)$ adesso quando ritorno anche per quando ritorno per le vacanze in Italia e: uhn: e: rivedo questo seminario e $(++)$ e: ormai rimasto quasi vuoto e:: $\mathrm{mi}$ mi viene una

230 stretta al cuore perché ai nostri tempi vederlo così rigurgitante pieno di vita e tante belle speranze in 
235 cose così cambiate ehn

tanti giovani e uhn: uhn: pieni di idealismo e: entusiasmo e adesso ridotto così ai minimi termini si può dire e: e è triste ri/ RItornare e:: uhn vedere le doc.: e lei attribuisce a:: e:: questa questa magari forse non l'ubbidienza alla chiamata

inf.: [ èh

240

doc.: ahn è a quali fattori secondo lei che cosa fa con che le persone con chi: i ragazzi non vadano più non si interessino?

inf.: [ sì: bene: certamente che:: $(+)$ certamente sono vari fattori che entrano in campo eh quando si entra in seminario a:: così piccolini a tredici anni come sono entrato io è: non si può proprio parlare ancora di vocazione $(+)$ e poi c'è tutta una strada c'è tutto un percorso da fare èh: per cui uno va preparandosi in tutti i sensi con lo studio e anche con la riflessione per sentire se è proprio questa vita eh: che vuole abbracciare

250 se è questa missione che vuole compiere se è questa insomma la vera vocazione $(+)$ e questo è una è una: la risposta non viene subito così da un giorno all'altro viene viene $(+)$ viene poco/ a poco a poco tanto è vero che anche l'impegno poi l'impegno definitivo eh: eh: $(+)$ che si

255 assume $(+)$ eh: anche ufficialmente davanti alla chiesa davanti alla società non è subito nei primi momenti nei primi giorni e neppure nei primi anni eh: solamente: dovranno passare parecchi anni prima di di uno ah: sottoscrivere questo impegno $(+)$

260 solamente quando viene ordinato sacerdote o compie o compie la professione religiosa allora sì $(+)$ diventa un imPEgno si conferma una vocazione ( noi ) ne/ nella stra/ strada facendo mi ricordo siamo entrati in molti in seminario siamo entrati in: una sessantina ma: siamo

265 arrivati in pochi alla fine a: alla all'ordinazione all'ordinazione siamo arrivati in ventidue $(+)$ in ventidue e poi in diciotto siamo partiti per le missioni

doc.: e: e la sua famiglia $(+)$ qual è stato per esempio il comportamento della sua fami:glia in confron:to con la 270 sua decisione per esempio di andare $(++)$ in seminario? inf.: (veda:) mia famiglia essendo io il primogenito $(+)$ eh: la 
mia famiglia avrebbe avuto piacere che io rimanessi $(++)$ che rimanessi in: famiglia che io $(+)$ rimanessi a ser/ a servizio della famiglia $(+)$ e:: $(+)$ (però) mi ha lasciato molta libertà non posso dire $(+) \mathrm{mi}$ ha lasciato molta libertà $(+)$ di scegliere la mia strada $(+)$ e:: di fare:: è un problema che forse quello ((si ascolta un grande rumore)) di rispettare

solo quello che era il mio desiderio $(+)$ di fare quello che io mi sentivo $(+)$ di più di: portato a: a fare: ha rispettato la mia

280 famiglia ha rispettato profondamente la mia decisione $(+)$ però io sapevo che se $(+)$ che il suo desiderio $(+)$ di mio padre per esempio sarebbe stato quello che io rimanessi $(+)$ con loro $(+++)$ che anche questo è è un problema $(+)$ è un problema:: non è facile a capire cosa vuol dire il distacco $(+)$ completo dalla famiglia $(+)$ per $(+)$ per una causa così per $(+)$ per la missione da compiere $(+)$ e per una vita consacrata $(++)$ eh è un problema che forse $i$ lai/ $i$ laici fuori che

sono nel mondo non $(+)$ riescono a capire a valutare dovutamente che cosa significa questo sacrificio questo

290 distacco questa $(++)$ questo impegno insomma l'impegno impegno di questa vocazione che porta conseguentemente a questo distacco

doc.: quindi di maniera che lei non si è mai dedicato allora a un'altra attività:: ha entrato e ha continuato

295 inf.: [ sì sì io sono

entrato in seminario a tredici anni $(+)$ ho continuato in se/ e la:: $(+)$ e:: tutti gli studi in seminario $(+)$ e:: $(+)$ fino all'ordinazione $(+)$ e:: tutto il curriculum vitae necessario da percorrersi e poi: come dicevo inizialmente $(+) \mathrm{mi}$ ha mi è stato chiesto se accettavo questa missione in Brasile io ho accettato $(+)$ sono venuto e qui ancora mi trovo

doc.: e a che ordine lei appartiene?

inf.: l'ordine: a: le al: qua: al quale io appartengo è la è: uhn:: la congregazione dei missionari di San Carlo (+) eh: o Scalabriniani $(+)$ eh: fondata giustamente per l'assistenza agli italiani emigrati $(+)$ ancora alla fine 
del secolo scorso $(+)$ milleottocentoeottantasette quando c'erano quelle grandi emigrazioni di massa allora:

Scalabrini ha sentito il bisogno di fondare una congregazione per dar assisTENza (+) spirituale e:: moRAle e e:: in: in: in quanto possibile anche: sociale e e alla a:gli emigranti agli emigranti

doc.: se lei fosse per esempio se lei fosse in Italia $(+)$ su

315 quali aspetti $(+)$ sarebbe diversa la sua vita sacerdotale immaginando per esempio così che: gli aspetti politici economici sociali influiscono nella sua nello svolgimento della sua vita sacerdotale?

inf.: eh se io fossi in Italia $(++)$ certamente là ah questa attività: che io compio qui questa attività ahn:: diciamo: di ordine spirituale questa attività PAStorale $(+)$ a:: servizio della chiEsa a servizio $(+)$ della $(++)$ del popolo di Dio a servizio della comunità cristiana eh:: certamente sarebbe: sarebbe differente $(+)$ in Italia ci sono: le parrocchie

325 eh: territoriali che costituiscono la: la struttura essenzialmente

della chiesa $(+)$ anche qui ci sono $(+)$ eh:: (ma qui) diciamo è:: è una forma un po': differente la legge il diritto canonico la legge della chiesa la struttura essenziale è uguale dappertutto in tutta la chiesa

330 universale $(+)$ però in ogni posto in ogni luogo $(+)$ in ogni paese $(+)$ e:: si (fa/) assume: aspetti differenti perché $(+)$ e: la chiesa cerca di adattarsi sempre alla realtà della della $(+)$ nazi/ del luogo $(+)$ della gente dove si trova $(+)$

335 doc.: sì

inf.: infine:: adesso per esempio in questo senso della nostra $(+)$ della missione della finalità della nostra congregazione $(+)$ in nel sen/ nel senso in vista della nostra missione si

340 può dire che $(+)$ è: molto: flessibile perché $(+)$ per esempio come stavamo dicendo $(+)$ eh:: in Italia $(+)$ in Italia ci si ci sono state queste queste grandi emigrazioni alla fine del secolo scorso e inizio $(+)$ ah $(+)$ e poi c'è stata la prima guerra mondiale $(+)$ poi col 
fascismo è è chiusa/ si è chiusa l'emigrazione $(+)$ poi è venuta la seconda guerra $(+)$ e dopo la seconda guerra ecco un'altra

l'ult/ l'al/ l'al/ l'ultima leva di emi/ di emigrazione (+) eh: che $(+)$ e quindi è stato fino al sessantacinque si può dire $(+)$ l'Italia è stata $(+)$ ah: è stata una nazione di $(++)$ emigrati emigrati di emigrati no? $(+++)$ e adesso doc.: uhn uhn

a prendere i sacchi adesso si $(+)$ avvera il contrario $(++)$

inf.: eh qui in Brasile era un paese di immigrazione dove venivano non solo dall'Italia ma da tante da tante parti del mondo da:: dagli altri stati eh: d'Europa poi anche ultimamente dall'Asia dai paesi asiatici dal Giappone della Corea $(+)$ e: eccetera $(++)$ adesso succede il contrario $(++)$ che c'è un riflusso $(+)$ perché il Brasile come io dicevo prima ha: che ha susci/ che ha suscitato tante speranze che era il paese del futuro a un certo momento ha incominciato a disilludere eh: la gente $(+)$ ha incominciato

(+) ahn:: far perdere la speranza soprattutto alla gioventù e quindi abbiamo notato abbiamo notato (+) ahn::

questo fenomeno che qui stesso in Brasile (dei) giovani

370 cercavano di: in tutti i modi eh: un cammino: di: differente cercavano in in poche parole di uscire di qui $(+)$ eh in cerca di nuove speranze e e succede che $(+)$ in altri paesi $(+)$ e nei paesi distrutti dalla guerra $(+)$ dove sembrava che tut/ che non ci fossero più: possibilità

375 a un certo momento eh: le cose si sono cambiate eh:: è arrivato il progresso è arrivato uhn:: dei

( ) si sono si sono si sono sommati si sono messi insieme dei fattori che hanno portato nuova vita nuova speranza nuovo progresso e: e questo: in generale 380 in Europa (+) in Giappone (+) eh:: i paesi asiatici Corea eh: in Australia in Canada eccetera e quindi molti giovani molti giovani si son/ brasiliani si sono diretti verso questi paesi e soprattutto giovani delle uhn:: delle:: figli di di antichi immigranti venuti da quelle 385 parti cosa fa ha cercato di $(+)$ sia per un richiamo delle radici $(+)$ sia per un prestigio della de de de delle 
nazioni dei dei loro no:nni dei loro an::/ antepassati e sia perché avevano perduto le speranze qui e in: questo in questo paese eh: che ancora è un grande paese e è successo questo fenomeno che abbiamo visto molti figli dei nostri dei nostri emigrati eh: ritornare cercare ogni modo/ in ogni modo di ritornare a: alle: loro origini e::: e:: di: $(+)$ di costruire una nuova vita in questi: in questi posti nèh? da dove sono partiti i loro antepassati oppure di tornare in questi paesi per fare qualche cosa qualche creare nuove possibilità anche per per cul/ per motivi culturali senza dubbio $(+)$ o forse anche per avventura $(+)$ e o magari per fare quattro soldi per poi eh: qui potere: costruire una casa poter mettere su qualche cosa di di:: di più stabile di più solido ahn: qui:: in Brasile stesso $(+++)$ quindi voglio dire che $(++)$ che questo sotto questo punto di vista ah: diciamo $(+)$ di ritorno è è:: un un fenomeno è un fenomeno molto interessante ah:: offerto agli agli studiosi nèh? di vedere come mai ques/ come dice Giambattista Vico corsi e ricorsi della storia come le cose si:: si girano si:

doc.: girano girano e si ripetono poi inf.:

[ appunto si riPEtono e: si ritorna alle fonti e::: si: e: si rinnovano anche no? è una cosa molto interessante $(+)$ questo fenomeno delle delle/ del riflusso del ritorno $(t)$ abbiamo visto anche adesso però abbiamo visto adesso per esempio a: a riguardo del terremoto del Giappone no? a Kobi eh:: dove c'erano molti di questi oriundi e: già: nisei sansei

415 che: (hanno) cercato una nuova speranza da dove erano partiti i loro antepassati il Giappone: che dicono che là si guadagna bene ((interruzione)) questi giovani che sono eh che sono partiti per per per il Giappone $(+)$ eh: giovani e anche $(+)$ meno giovani in cerca di una forTUna 420 o: per rimanere là o:: per ritornare poi con un po' di soldi e qui poter fare qualche cosa cosa succede? improvvisamente viene questo terremoto $(++)$ e allora ah: benedetto il Brasile si sono ricordati abbiamo visto quando sono arrivati qui di ritorno in Brasile come si 425 sono perfino inginoCCHlati a baciare questa terra $(++)$ quindi ve/ vedi come tutto è relativo $(++)$ mai si può dire 
ecco abbiamo rice/ abbiamo raggiunto il paradiso qui in terra: non c'è il paradiso qui in terra $(++)$ anche nei anche i luoghi più:: più: famosi più:: progrediti più benedetti èh:: a un certo momento possono $(+)$ possono $(+)$

disilludere $(+)$ o per un motivo o per un altro $(++)$ quindi diciamo: eh:: cerchiamo di di non essere troppo esigenti troppo: idealisti l'essenziale è di/ sapersi accontentarsi di quello che Dio ci dà e:: saper valorizzare nel posto dove siamo gli aspetti positivi delle persone $(+)$ degli avvenimenti $(+)$ eh: delle cose doc.: considerando la situazione brasiliana su tutti gli aspetti quale dovrebbe essere secondo lei la preoccupazione maggiore della chiesa?

440 inf.: la preoccupazione maggiore della chiesa (+) è:: senza dubbio in primo luogo sempre è la sua missione spirituale l'aspetto spirituale $(+)$ ahn:: ahn della chiesa stessa e della: noi vogliamo sempre $(+)$ noi vogliamo sempre $(+)$ in primo luogo ah: $(+)$ la nostra missione nel nel senso di:

445 di non perdere di vista $i$ valori: essenziali i valori eterni $(+)$ ecco e poi in quanto possibile anche: eh anche: l'aspetto: temporale senza dubbio perché $(+)$ non si può dividere l'essere umano in: spirituale e materiale è un'unità unica e:: che deve essere portata alla: alla 450 crescita e: alla felicità e alla salvezza eterna e quindi c'è in in quanto possibile eh: la chiesa soprattutto qui in Brasile si interessa molto anche per l'aspetto sociale (+) delle comunità dei problemi sociali: dei: uhn:: dei delle delle ma/ dei dei cammini dei modi da usare per a:: per a:iut/ per collaborare in questo senso collaborare per per il

progresso eh:: in tutti i sensi anche il progresso scientifico il progresso umano il progresso sociale e: uhn:: e: tanto è vero che $(+)$ che sempre si parla nelle nostre riunioni ecco cosa fare come fare $(+)$ per aiutare il popolo

460 a risolvere questi problemi $(+)$ dell'educazione della sanità e:: della:: della moradia o come si dice in italiano $(+)$ della abitazione e:: $(+)$ del lavoro $(+)$ del del dei problemi della disoccupazione? e così via si interessa molto la chiesa oggigiorno della della parte sociale (+) 
465 ANzi qualcuno: qualcuna CRItica:: perfino critica molto la chiesa perché si è messa troppo nel sociale $(+)$ perdendo di vista un po' quella che era la sua $(+)$ missione e:essenziale che che è:: soprattutto spirituale di ordine spirituale

470 doc.: questa parrocchia che le/ svolge qualche attività: assistenziale?

inf.: [ no:: questa nostra parrocchia la Madonna della Pace proprio qui in CENtro di San Paolo è: oltre che essere parrocchia territoriale $(+)$ cioè ha un suo territorio

475 piccolo territorio come tutte le parocchie hanno $(+)$ doc.: uhn uhn

inf.: oltre che essere parrocchia territoriale territoriale è anche parrocchia personale degli italiani $(++)$ cioè ah:: raggiunge tutti gli italiani di San Paolo $(+)$ i suoi $i$

480 suoi/ le sue divise/ i suoi confini non sono $(+)$ materiali confini materiali sono confini così di ordine: eh:: teorico di ordine: psicologo eh:: e per per questo si chiama parrocchia perSOnale o anche chie/ come è chiamata

$(+)$ parrocchia nazionale cioè della della $(+)$ parocchia della comunità italiana della nazione italiana di San Paolo $(+)$ poi oltre che essere parrocchia personale degli italiani $(++)$ eh:: e anche: $(+)$ anche allo stesso allo stesso livello eh:: anche la responsabilità e la cura degli dei: degli emigrati eh:: latino-americani latino-americani che come ho detto in primo inizialmente la nostra congregazione era è fonda/ è stata fondata per immigranti $(+)$ ma non si/ no: no: inizialmente sì solo per gli immigranti italiani ma poi $(+)$ quando l'emigrazione italiana è finita in Italia e allora soprattutto qui in Brasile si è aperta per l'emigrante in generale e: prova sia che qui la nostra: chiesa della Madonna della Pace per dieci anni è stata sede anche della parrocchia coreana/ dei coreani e il parrocco coreano abitava qui con noi nella comunità della Pace qui coi sacerdoti della 500 Pace $(++)$ poi oltre a queste attività di ordine pastorale abbiamo anche abbiamo anche una: una casa di accoglienza $(+)$ ahn:: di:: per emigranti (in necessità) questa sarebbe una:: un aspetto della nostra ah:: del nostro lavoro 
sociale della nostra $(+)$ missione sociale nel campo della chiesa e in questa casa di accoglienza abbiamo più o meno sempre circa $(+)$ duecentoecinquanta persone alle quali diamo vitto alloggio a:assistenza giuridica e:: sociale spirituale e anche $(+)$ incamminamento alla/ a un lavoro $(+)$

510 responsabilità $(++)$ poi abbia:mo: tutte le altre attività comuni di altre parrocchie $(+)$ incontro: incontro delle coppie con Cristo abbiamo: catechismo abbiamo: la i: vari corsi necessari per il matrimonio per il battesimo per: la CREsima e così via

515 doc.: per queste: per questo tipo di assistenza eh:: ci vogliono anche delle:

inf.: [ sì dei mezzi

doc.: dei mezzi appunto queste associazioni italiane per esempio loro hanno:: non so: aiutano ( )

520 inf.: sì: noi abbiamo sempre avuto aiuti abbiamo in primo luogo $(+)$ ah: la fiducia nella divina providenza no: che sempre ci aiuta poi abbiamo la struttura parrocchiale $(+)$ poi abbiamo sempre $(+)$ sempre avuta: abbiamo ancora dei benefattori specialmente nella comunità italiana che:

525 fin da principio hanno aiutato: nella: nella comunità italiana e: che poi: magari hanno fatto fortuna e che: dicono come noi siamo stati aiuTAti $(+)$ quando siamo arrivati QUI $(++)$ e la maggior parte sono arrivati (+) eh: POveri no? senza nulla come no/ come noi siamo stati 530 aiutati $(+)$ quando avevamo quando abbiamo bussato qui alle porte $(+)$ della casa brasiliana $(+)$ con le mani in mano $(+)$ come queste porte ci sono state aperte con tanta generosità $(+)$ e abbiamo trovato qui in Brasile questa accoglienza così grande $(+)$ così anche noi adesso:: in

535 quanto possiamo: cerchiamo di aiutare i nostri fratelli che si trovano nelle stesse circostanze nostre quando siamo arrivati e forse anche in difficoltà maggiori $(++)$ per cui eh:: questa diciamo $(+)$ opera $(+)$ di:: fraternità e anche uno strumento di: di integrazione: e: di

540 solidarietà cristiana $(+)$ senza dubbio $(+)$ un esempio una na na una necessità questa in/ questa integrazione questa solidarietà cristiana è una: è un è un è un motivo è un 
ideale è un $(+)$ e un ( ) che che:: insomma che che fa forza che fa: nascere nuo/ nuovi ideali

545 nuo/ nuove eh:: espe/ esperienze e:: e nuove anche nuove inspirazioni specialmente nella gioventù: che hanno bisogno di un ideale nella vita no? non solo qui in Brasile ma in tutte le parti del mondo vediamo sorgere iniziative di volontariato di: di integrazione di

550 solidarieT $\grave{A}$ e: eccetera $(+)$ specialmente specialmente nel diciamo in questo: campo emigratorio $(+)$ di: molta gente $(+)$ che arriva che parte eccetera eh: c'è bisogno di questa solidarietà di questa comprensione di questo rispetto ai vari motivi alle varie cause che: che: fanno: che che $(+)$ che producono questo fenomeni/ questi fenomeni

$(+)$ quindi vediamo vediamo che $(+)$ che:: è difficile è difficile diciamo dire eh: no/ noi non abbiamo di bisogno: vediamo che dovuto a questo questo diciamo a questa: a questi fenomeni eh: come sia

560 importante questa solidarietà $(+)$ abbiamo detto in principio

che in in Italia era paese di emigrazione $(+)$ adesso è diventato paese di immigrazione molti che $(+)$ là ritornano $(+)$ tu hai visto quando sei stata là $(+)$ come: come ci siano molti contrari anche a questa $(+)$ a

565 ricevere questi questi:: extra/ extra-europei come si chiamano doc.: extracomunitari

inf.: extracomunitari $(+)$ eh: questi stranieri come eh: specialmente quando sono quando quando arrivano da certi:

da certi posti da certe da certe:: ahn:: etnie: molto

570 differenti dalla nostra dalla nostra formazione come sono i musulmani e eccetera e:: e ci vuole mol:ta ci vuole molta comprensione molta pazienza $(+)$ perché: $(+)$ perché sennò si formano dei dei terribili confronti e: anche assassinati e: criminalità eh: per motivi razzisti per

575 motivi: così preconcetto eh: eh: dopo l'espe/ le tristi esperienze de de delle guerre che noi abbiamo fatto $(+)$ è:: è necessario $(+)$ ah portare i giovani a una a una a una a un livello di: di formazione: diciamo così elevato da passare SOpra questi (+) questi questi pregiudizi eh: 
580

questi: eh: questi motivi di divisione eh: la solidarietà umana e cristiana sempre $(+)$ sempre è stato un valore importante nella chiesa e sempre sarà un $(+)$ un grande: un grande/ uno strumento di lavoro un grande: ideale nella vita e sempre porterà buoni frutti

585 doc.: allora vediamo un po' o::oggigiorno lei per esempio lei frequenta in uguale quantità eh brasiliani e italiani?

inf.: sì oggigiorno io ahn: $(+)$ io frequento ahn: con la stessa diciamo $(+)$ simpatia con la stessa $(+)$ al: diciamo $(+)$ con la stessa gioia e entusiasmo sia l'ambiente italiano e come come l'ambiente brasiliano mi trovo mi trovo bene: in:

ambedue le parti abbiamo abbiamo MOLte attività italiane e:: $(+)$ qui anche fuori eh: frequento $(+)$ spesso mi trovo bene con loro e mi chiamano mi invitano e:: così anche nella nostra attività $(++)$ parrocchiale nostre attività

$595 \quad(+)$ con la $(\quad)$ con i brasiliani pure: $(+)$ eh mi sento molto bene mi trovo bene con loro $(+)$ e si può dire che $\mathrm{mi} / \mathrm{mia}$ la mia vita il mio lavoro è diviso così in:: ambedue le $(+)$ le le parti ambedue le nazionalità però sempre senza creare senza creare $(+)$ ah: 600 ghetti nazionalismi campanilismo $(+)$ eccetera sem:pre: in vista dell'integrazione della fraternità $(+)$ sempre rispettando sì le differenze perché $(+)$ sempre le differenze ci sono ma: MAI ah: uhn: facendo leva sulle differenze per creare divisione ma giustamente il

605 contrario sommare sommare le differenze per una una ma/ ma/ maggiore arricchimento ah: della comunità e:: e per: ah: una maggiore: unione e solidarietà fra i popoli doc.: e con gli italiani quando lei è insieme agli italiani parlate sempre in italia:no::?

610 inf.: [ sì in generale sì quando siamo quando siamo con gli italiani parliamo in italiano anzi eh: si può dire che $(+)$ c'è una soddisfazione in certo qual senso di:: rispettare di caPIre anche gli stessi dialetti delle varie regioni

615 doc.: ( ) perché ancora è molto parlato il dialetto forme [ è sì ((ride)) perché ci sono ancora eh eh eh queste caratteristiche proprie di di esprimersi $(+)$ di 
comportarsi attraverso la la tradizione de/ della che ciascuno ha portato e dei dialetti una delle tradizioni

620 delle forti caratteristiche delle regioni italiane sono i dialetti tanto è vero che è diFFicile a volte capire questi dialetti $(+)$ per esempio noi del nord abbiamo delle: ahn:: per noi del nord per VAri motivi non solo per i dialetti è:: ci è è difficile eh capire quelli del

625 sud dell'Italia perché c'è sempre stato un po' di campanilismo di di: come si dice eh: $(+)$ tra il sud e il nord d'Italia sempre c'è stato una:

doc.:

inf.: eh eh eh una specie di eh: eh: una specie di: (+) antitesi di: eh

630 doc.: di confronto

inf.: di confronto sì di confronto di preconcetti eccetera (+) ancora ci sono anche Italia stessa ma però qui: ha: notato che qui per esempio a San Paolo eh:: non non si:: questo questo questo confronto non c'è $(t)$ qui siamo

635 tutti italiani tutti fratelli tutti uniti ci rispettiamo gli uni con gli altri ognuno con le sue come dicevo prima ognuno con le sue caratteristiche con le sue tradizioni $(+)$ facendo di queste differenze una ricchezza per per esempio qui nella chiesa della Pace facciamo facciamo 640 ogni ogni ogni prima domenica del mese u:una messa per gli italiani $(+)$ e ogni ogni prima domenica del mese eh:: questa messa è patrocinata da una regione differente eh: quindi si approfitta che ognuno venga con le sue caratteristiche con la sua maniera di essere con le sue

645 tradizioni anche per offrire: una una diversità una novità agli altri che magari non conoscono queste differenze $(+)$ queste tradizioni queste ricchezze una di queste una di queste differenze che ahn: che costituiscono diciamo la diversità del popolo italiano

650 sono questi dialetti son/ sono questi linguaggi differenti MOLto differenti uno dall'altro $(+)$ perché bisogna sapere che bisogna ricordare che l'Italia $(+)$ fino a fino alla fine del secolo scorso era divisa in tanti stati $(+)$ in tanti stati indipendenti uno dell'altro $(+)$ e quasi si 655 può dire che ognuno aveva sua lingua propria $(+)$ e la lingua nazionale e la lingua nazionale $(+)$ italiana 
ufficiale italiana è sorta $(+)$ si può dire con: con l'unità d'Italia $(+)$ e poi è stata molto propagata attraverso la televisione eccetera $(+)$ adesso in tutte le parti d'Italia certamente eh: si si parla bene l'italiano nelle città tu hai visto nèh? però:: eh:: in un primo tempo $(+)$ soprattutto per causa della televisione che aveva dato $(+)$ enfasi a questa a questa a questa lingua uffiClAle eh:: in certo qual senso erano disprezzati i

665 dialetti ade:sso eh:: succede il contrario si va in cerca di: di: di non di non dimenticare di di di rifare di:: ahn:: di:: studiare di nuovo di di riportare in vita questi dialetti perché sono considerati una ricchezza una ricchezza anche culturale $(+)$ che non si può: così::

670 doc.: disprezzare

inf.: disprezzare: che non si può: dimenticare o: mettere tutto nel fuoco nel bruciare perché è come se fosse $(+)$ eh: eh: $(+)$ una: $(+)$ una cosa da $(+)$ da distruggere no eh: c'è questo

675 doc.: okay allora

inf.: [ adesso ( )

doc.: per lei $(+)$ ci sono degli argomenti speciali che le è più facile parlare in italiano o è più facile parlare in portoghese o comunque per lei non lei non sente qualche differenza lei:

inf.: ma adesso si può dire che dopo dopo quarant'anni che sono qui (+) eh: eh: insomma non non sento difficoltà non non non ho dimenticato l'italiano come succede a molti che vanno fuori di di della propria patria eh:: dopo un po'

685 di tempo quando ritornano non sono più capaci di ((ride)) di di parlare di parlare oppure incominciano uhn: tirar fuori $(+)$ parole esotiche parole strane che fanno ridere anche la gente no? ((ride)) no io quando sono con gli italiani cerco di parlare l'italiano $(+)$ ah: 690 sempre mi sono sforzato quando sono con i brasiliani cerco di di: parlare: eh: insomma il portoghese eh: senza difficoltà eh: insomma si sa che la che la comunicazione le espressioni il modo di espressarsi eh:: non è facile in:: in: qualsiasi maniera anche anche nella propria lingua anche nella propria patria eh:: sa esprimere $i$ sentimenti esprimere ehn:: i propri concetti le proprie 
idee è:: sempre è una: $(+)$ è una sfida per tutti no (+) una una $(+)$ eh: e quindi eh:: sì difficoltà ci ci sono () trovo ancora alle volte un po' di difficoltà nell'esprimermi eh

700 però è: una difficoltà: comune che tutti quanti sentono che tutti quanti hanno anche a volte per dire una cosa semplice allora si dice ma come devo fare come devo dirglielo co:/ come devo esprimermi come posso dire (+) non è vero?

doc.: sì sì

705 inf.: perché:: questo è naturale

doc.: è naturale sì

inf.: èh

doc.: lei si sente $(+)$ brasilianizzato o conserva ancora delle abitudini italiane tipo alimentari o delle tra/ dei

710 costumi

inf.: sì conservo ancora conservo ancora delle abitudini italiane sì non posso $(++)$ non posso: negarlo $(+)$

doc.: ahn non fa a meno alla pastasciutta ehn?

inf.: appunto ((ride))

715 doc.: okay e lei viaggia sempre per l'Italia? con quale frequenza? lei ha detto che ( )

inf.: [ eh: sì viaggio viaggio in Italia $(+)$ eh: ogni tre anni $(++)$ ogni tre anni è: è sempre un piacere rivedere la nostra patria $(+)$ vedere confrontare eh:

720 l'evoluzione degli avvenimenti del progresso di di vari popoli mi è sempre piaciuto per esempio la storia (+) comparata eh:: ahn: eh: tra un: eh: in tutti i sensi uhn: tra le genti tra i popoli storia comparata $(+)$ uhn: sui punti di vista culturale sociale e anche spirituale ora

725 per esempio per me che sono venuto subito dopo la guerra per dire come era il Brasile adesso $(+)$ e ora da in quel tempo e (a) vederlo come è adesso $(+)$ confrontando con l'Italia $(+)$ com'era dopo la guerra $(+)$ e: come è adesso è è è insomma è uno studio interessante non è vero?

730 doc.: ( )

inf.: perché in quarant'anni le cose sono cambiate molto sia qui come là e in che senso? e perché? e come? e questo eh:: quindi avendo la possibilità di ritornare ogni: tre anni (+) eh:: mi è possibile sempre fare questa (+) 
rapporto questo confronto questo rapporto questo si chiama questo esame questo studio (ma) non tanto in profondità insomma

anche così a occhi visti si vede si si vedono gli effetti le differenze e: poi anche per rivedere la famiglia mi piace ri/ ritornare perché qui in Brasile non ho nessuno

740 doc.: lei ha parenti

inf.: [ $\mathrm{i}$ i miei pare/ no qui in Brasile non ho: non ho la mia famiglia non è non è venuto nessuno dei miei familiari qui in Brasile $(+)$ eh: quindi anche un rivederci è è un dovere e finché ci sono i genitori

745 vivi è è è un dovere ancora più santo sacrosanto più più speciale no? poi quando vengono a mancare i genitori certo che si sente meno questo attacco/ questo affetto però lo si sente ancora i fratelli i nipoti le nuove gene/

750 i nuovi che vengono su

le novità nella famiglia continuano sempre ( ) noi italiani abbiamo un grande attaccamento alla famiglia $(+)$

la famiglia per noi è una cosa molto importante la famiglia la casa $(++)$ eh: è una realtà molto sacra

755 molto rispettata molto molto vissuta eh: eh: almeno in Italia nèh? in ques/ in questo senso $(+)$ forse più di qui $(++)$

doc.: uhn uhn è vero lei $(+)$ tanto per finalizzare inf.: eh

760 doc.: lei potrebbe raccontare un episodio importante eh: della sua vita che stia legato qui al Brasile inf.: un episodio importante?

doc.: uhn

765 e certamente ((tosse)) inf.: nella mia vita $(+)$ che sia legato qui in Brasile (+) eh: accettare accettare la proposta di venire in Brasile l'aver accettato questa proposta $(+)$ capisco sempre di più che sia stato $(+)$ l'episodio più importante della mia vita

770 ah: di missionario di della mia vita di emigrante anche che mi son fatto emigrante con gli emigranti e conseguentemente 
doc.: [ cerca di di confrontare

inf.: non è vero? quindi per me io penso che il fatto di essere venuto qui in Brasile eh: questo sia stato avendo accettato il Brasile della mia vita qui in Brasile (+) perché poi il resto sono decorrenze sono conseguenze eh: che sono venute con questa decisione $(+)$ il posto eh: i cambiamenti le varie persone $i$ vari avvenimenti anche qui in Brasile stesso uhn: in tutti $i$ sensi politici sociali eh: sconvolgimenti avvenimenti buoni anche tutto il progresso raggiunto ahn: ecco qui c'è una cosa per esempio che c'è $(+)$ che c'è una novità una caratteristtica che c'è qui che si trova qui in Brasile e che si trova in Europa è questa che là si dice che è tutto fatto adesso ormai è c'è stata quella ricostruzione c'è stata la guerra ma sempre su sui ( ) antichi sia pure in senso moderno però lì sempre tutto fatto ormai no? invece qui in Brasile eh: c'è c'è c'è stato uno sviluppo una evoluzione eh: eh: c'è ancora uno sviluppo un'evoluzione che: che cambiano le cose da da un anno all'altro si si va in un paese non è più come prima in un paese in una città in un posto eh: tutto eh: si trova cambiato (+) quello che non succede ( ) io vedo quando vado in Italia ci sono molti cambiamenti però i paesi sono ancora quelli: le tradizioni i costumi eccetera l'avvenimento più importante eh: in riferenza anche al Brasile

e poi se si si dovesse dire episodi episodi così diciamo così di novità di eh: questi succededono ogni o/ o/ ogni momento ci sono qui in Brasile ci sono quindi molto molto abbondante mo/ molto $(+)$ di di varia natura bisogna 805 vedere cosa che tu anche intendi co/ come episodi che $\mathrm{mi}$ abbi/ abbiano marcato di più qui in Brasile e in che senso

doc.: [ qualche fatto particola:re:

inf.: tu sai che qualche fatto particolare alle volte possono essere piccole piccole cosettine piccole cosettine che 
Inquérito 7 (DI).

810

doc.: sì

rimangono impresse nèh? più di cose grandi che magari agli altri non dicono nulla ma che

per me sono sono importanti

inf.: ma che perfino mi pare che sia che sia

815 un po' banale ((ridendo)) raccontare queste queste piccole cosettine che magari agli altri chissà come

potranno $(+)$ eh: ricevere come potranno interpretarle (come potranno eh:) capisci?

doc.: sì sì 